\title{
Extraction and Enrichment of Copper by Liquid Emulsion Membrane Using LIX 664N
}

\author{
Rashmi Kumar1, Dahyalal J. Shah², Krishna Kant Tiwari ${ }^{3}$ \\ ${ }^{1}$ D. J. Sanghvi College of Engineering, Mumbai, India \\ ${ }^{2}$ Mukesh Patel School of Technology Management \& Engineering (NMIMS), Mumbai, India \\ ${ }^{3}$ Jaypee University of Engineering and Technology (Guna), Madhya Pradesh, India \\ Email: rashmikumardjs@yahoo.co.in
}

Received 14 October 2014; revised 9 November 2014; accepted 5 December 2014

Copyright @ 2014 by authors and Scientific Research Publishing Inc.

This work is licensed under the Creative Commons Attribution International License (CC BY). http://creativecommons.org/licenses/by/4.0/

c) $\underset{\mathrm{EY}}{\mathrm{C}}$ Open Access

\begin{abstract}
Process intensification using liquid emulsion membranes (LEMs) for extraction of copper has been studied in this work. In LEM process the extraction and stripping processes are combined in one stage. The solubility of the solute or the chemical affinity between the solute and carrier reagent in the membrane results in the separation of the solute from the feed mixture. In this work the membrane phase comprised of LIX 664N as the extractant dissolved in kerosene and Span 80 as surfactant. The stripping phase was sulfuric acid with $180 \mathrm{~g} / \mathrm{l}$ concentration and feed phase had copper concentration of $3.5 \mathrm{~g} / \mathrm{l}$. Effect of various process parameters such as batch contact time, speed of agitation, $\mathrm{W}: 0$ ratio and treat ratio has been experimentally investigated to get better insight of the process. The maximum enrichment of copper in the internal phase obtained was 11.6 times with batch contact time of 10 minutes, agitation speed of $300 \mathrm{rpm}$ and treat ratio of 6:1.
\end{abstract}

\section{Keywords}

Extraction, Enrichment, Liquid Emulsion Membranes, LIX 664N, Copper

\section{Introduction}

Solvent extraction has been effectively used to remove and recover heavy metals from dilute effluents. Enrichment by solvent extraction depends on the maximum loading point of the extractant. Extractants for copper extraction are like LIX reagents, ACORGA, Kelex, D2EHPA, etc. Compared to solvent extraction, liquid emulsion membranes (LEMs) have several advantages. LEMs can acccomplish both extraction and stripping in one step. LEMs remove the equilibrium limitations of solvent extraction by combining extraction and stripping in a single operation, thereby achieving reduction of metal concentration in the feed stream to very low levels. LEMs 
can be used for high enrichment of metals from dilute streams as compared to solvent extraction. The small size of the globules provides high interfacial area of mass transfer. Thus compared to the conventional solvent extraction process the requirement of solvent inventory is low and mass transfer rate is high for LEMs due to availability of large surface area for extraction. Process intensification is possible by using LEMs which would help in reducing the operating cost. Also the size of equipment is smaller. $40 \%$ investment savings for liquid membrane can be made as compared to solvent extraction [1]. In addition to the equipment-related advantages, there are additional advantages of using the LEM technology. They lie primarily in the amount of crud that is formed and organic losses associated with extraction and crud formation [2]. The first large scale plant with a throughput of $75 \mathrm{~m}^{3} \cdot \mathrm{h}^{-1}$ was built for the Austrian viscose industry for the recovery of zinc from spin baths [3].

In principal, the liquid emulsion membrane processes are a three-phase dispersion system, where primary emulsion consisting of organic and stripping phase is dispersed in the feed or effluent phase, which is the phase to be treated. The strip phase is encapsulated in the organic membrane phase to form water in oil (W/O) type of emulsion. The emulsion globules are then dispersed in the aqueous feed phase to form W/O/W phase. Membrane phase refers to the phase that separates the encapsulated phase in the emulsion and the external continuous phase. Each globule contains many droplets of encapsulated inner or receiving phase. The drop diameter varies between 0.1 and $2 \mathrm{~mm}$ and the diameter of the dispersed droplets varies between 0.5 and $10 \mu \mathrm{m}$ [3]. Therefore emulsion will produce large volume/surface ratios for very rapid mass transfer by extraction and the small size of internal droplets will provide high interfacial area for stripping.

For the separation of metals even in very low concentration, the carrier is an organic soluble extractant, which selectively combines with the solutes to form a metal-carrier complex. This complex will permeate through the membranes from the outer to the inner interface. On contact of the emulsion with the feed phase, the solute is transported through the membrane into the internal droplets of the stripping phase. At the inner interface, the complex decomposes by the reversal of the equilibrium reaction; the metal ion is liberated into the internal phase; and the regenerated carrier goes back into the interface of membrane phase to pick up the solute. After extraction the loaded emulsion is separated from the feed stream and demulsified to yield oil phase that can be recycled. Demulsification can be done by centrifugation, sedimentation, thermal breaking and electrostatic method. High voltage electric field is a very efficient method to demulsify emulsions. The metal concentrated in the receiving phase can be recovered by electroplating.

But there are some drawbacks of LEMs. One major problem in the development of emulsion liquid membranes is that on one hand the emulsion has to be very stable; and on the other hand the emulsion has to split in order to recover the concentrated inner phase [4]. Also water transport to the stripping phase and break-up of emulsion decrease the efficiency of the overall enrichment process. The globues of water-in-oil-in-water (W/O/W) membranes swell due to transfer of water from external aqueous phase to the internal water droplets [5]. Attempts to minimize these effects should be taken.

In this research work the objective of our study is to demonstrate the application of LEM technique for recovery and concentration of copper from very dilute solutions as LEM process provides process intensification and is better alternative to the conventional extraction process. The various process parameters have been studied to aid technology development for recovery of copper from waste streams with very low concentration. The parameters affecting the rate and extent of extraction as extraction time, volume fraction of internal aqueous phase, concentration of surfactant, optimum speed of agitation and volume ratio of the emulsion phase to external aqueous phase have been studied in order to have a better understanding of the dynamics of the LEM technique.

LEM application for recovery and enrichment of Nickel [6], Ruthenium [7], Rhodium [8] has been reported. There have been data on LIX 984N-C [9], LIX 54 [10], LIX 84 [11] for copper extraction by LEMs. In this sudy extractant LIX 664N has been selected based on the excellent extractive property of LIX 664N for copper by solvent extraction [12]. LIX 664N is a mixture of 5-nonylsalicylaldoxime with a proprietary ester modifier and a high flash point hydrocarbon diluent. Salicylaldoximes provide high copper transfer kinetics and extractive strength. Stripping is done by strong sulphuric acid (180 g/l).

\section{Apparatus and Procedure}

\subsection{Materials}

LIX 664N was supplied by BS International, Mumbai. Kerosene was taken from local market and the surfactant Span 80 (Sorbitan monooleate) was supplied by Mohini Organics (P) Ltd., Mumbai, as a free sample. Span 80 is 
a nonionic surfactant with a HLB value of 4.3 and a molecular weight of 428. All other chemicals were of AR grade. For preparing the feed solution of $3.5 \mathrm{~g} / \mathrm{l}$ of copper required amount of copper sulfate was dissolved in de-mineralized water.

\subsection{Experimental Procedure}

First water in oil emulsions was prepared with a three-blade impeller in a glass vessel. The membrane consists of varying proportions of surfactant Span 80 and carrier reagent LIX 664N in diluent kerosene. The internal strip phase of sulfuric was added dropwise into glass vessel containing the organic membrane phase. A predetermined ratio of organic membrane phase to the internal strip phase was maintained. The contents were stirred and emulsification done at 5000 by the hgh speed impeller for 10 minutes. The composition of the liquid membrane is crucial in determining the robustness and therefore many trial experiments were performed to obtain the best formulation. The emulsion stability was 70 minutes with $2 \%$ Span and was sufficient for the entire experiment to be completed. An excellect milky white and stable emulsion was obtained.

The emulsion was then dispersed in feed phase of copper salt from which copper was to be extracted. Extraction was carried in a baffled glass vessel of diameter $8.75 \mathrm{~cm}$ and height $12 \mathrm{~cm}$ using an impeller of 10 mm diameter and speed in the range of $300-500 \mathrm{rpm}$. pH of feed phase was maintained at 2 by adding $\mathrm{H}_{2} \mathrm{SO}_{4}$. Samples of about $5 \mathrm{~cm}^{3}$ were withdrawn at different time intervals from the aqueous feed phase and analysed for copper concentration. At the end, the emulsion was separated from feed phase by simple gravity separation in separating funnel and broken by heating to about $90^{\circ} \mathrm{C}$ to separate the internal strip phase from the membrane phase. The metal ion content in the feed phase was measured by AAS. The copper extracted into the inner strip phase was calculated.

\section{Results}

Extraction of copper by LIX can be described as:

$$
2 \mathrm{HR}_{\text {org }}+\mathrm{Cu}_{\mathrm{aq}}^{2+} \rightleftharpoons \mathrm{CuR}_{2 \text { org }}+2 \mathrm{H}_{\mathrm{aq}}^{+} \text {. }
$$

Here HR is the extractant and aq. and org. are the aqueous and organic phases, respectively.

$K_{e x}$, the extraction equilibrium constant for extraction of $\mathrm{Cu}$ with LIX can be represented by the following equation

$$
K_{e x}=\frac{\left[\mathrm{CuR}_{2}\right]_{\mathrm{org}}\left[\mathrm{H}^{+}\right]_{\mathrm{aq}}^{2}}{[\mathrm{Cu}]_{\mathrm{aq}}[\mathrm{HR}]_{\mathrm{org}}^{2}} .
$$

Stripping can be described as:

$$
\mathrm{CuR}_{2 \text { org }}+2 \mathrm{H}_{\mathrm{aq}}^{+} \rightleftharpoons 2 \mathrm{HR}_{\text {org }}+\mathrm{Cu}_{\mathrm{aq}}^{2+} .
$$

Extraction rate of copper from an aqueous phase is divided into following steps: 1) diffusion of copper ions from external aqueous phase to the external interface of the emulsion globule; 2 ) interfacial reaction between copper ions and LIX at the external interface; 3) diffusion of copper complex into the emulsion globule; 4) stripping of metal ions from the metal complexes by strong acid in the strip phase. This leaves free LIX in the membrane phase; 5) transfer of the free LIX to the external interface again to form a complex with fresh copper ions. Thus LIX shuttles between the two interfaces.

\subsection{Effect of Extraction Time on Extraction of Copper}

The extraction time or contact time is one of the most important parameter in LEM system. Kulkarni et al. (2000) [13] observed that longer residence time resulted in more transfer of water into the internal phase resulting in swelling of membranes which subsequently might have initiated breakage of the emulsion phase. This causes leakage of solute from the internal phase to the external phase. Similar results were obtained in the present study. But at the same time an appropriate extraction time is needed for maximum mass transfer of metal ions from the external phase to the organic membrane phase. Figure 1 exhibits the percentage extraction of copper from the 


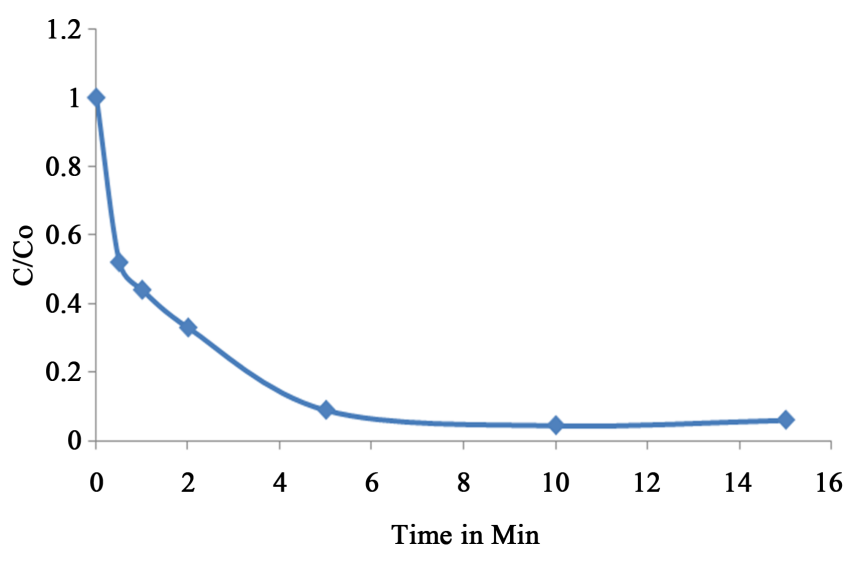

Figure 1. Effect of mixing time on copper extraction.

external phase as a function of extraction time.

The extraction performance increased when the extraction time is increased and reached 10 minutes. After 10 minutes, the degree of extraction decreased probably as a result of the swelling and subsequent breakage of the emulsion phase. This emulsion breakage causes leakage of extracted copper from the internal phase to the external phase and therefore, the metal concentration remaining in the external phase increased after 10 minutes of contact time. Thus, further extraction time will cause emulsion break-up. For further study total extraction time was selected as 10 minutes for LEM process as maximum enrichment of copper for 10 minutes.

\subsection{Effect of Volume Fraction of Internal Aqueous Phase on Extraction of Copper}

Internal stripping phase volume has large influence on extraction of the metal. With increased stripping phase volume, the emulsion globules are more densely packed with stripping phase droplets. This results in decrease in diffusion path length for copper-LIX complexes before getting stripped. Hence increase in striping phase volume is analogous to thinner membranes. The increased internal droplet diameter increases the stripping capacity. But very large volume of internal reagent is harmful to stability of membranes.

It is observed from Figure 2 that with increase in the volume fraction of W:O ratio from 3:7 to 1:1 the extent of extraction increased. With internal phase volume as $30 \%$ of the total membrane phase volume, the extraction\% was $94 \%$ and for $50 \%$ volume, it was $97 \%$. This increase can be attributed to the increase in capacity of the internal phase to strip copper from the membrane phase resulting in increase in mass transfer rate.

\subsection{Effect of Stirring Speed on Extraction of Copper}

Hydrodynamics play an important role in the performance of LEMs apart from the components. Speed of agitation affects the rate of transfer of solute through the liquid membrane. Theoretically with increase in agitation speed smaller sized globules are formed leading to increase in interfacial contact area between external and membrane phases and an increase in the mass transfer coefficient. But very high speed beyond certain critical level of agitation, can lead to swelling and rupture of the emulsion globules. This breakage of emulsion droplets reduces the overall enrichment and extraction. Therefore a suitable optimum speed has to be found that would give best results.

Few initial trials showed that minimum $200 \mathrm{rpm}$ was necessary to keep the emulsion uniformly in dispersed phase. As seen from Figure 3 as stirring speed was increased from 300 to 400 rpm the extraction of copper increased. But further increase of speed to $500 \mathrm{rpm}$ decreased extraction of copper. As the speed increased from 300 to $400 \mathrm{rpm}$, the emulsion globules are uniformly distributed in the continuous phase and smaller globules are formed thereby increasing the membrane surface area and hence the mass transfer rates. Further increase of speed to $500 \mathrm{rpm}$ causes some particles to be broken because of shear and increased swelling. The swollen particles can breakdown on their own after reaching a larger size or breaking is induced by shear. At $500 \mathrm{rpm}$, high breakage indicated less extraction and enrichment. Thus the extraction performance was best in the range of 300 - 400 rpm as the mass transfer rate is faster than break up. 


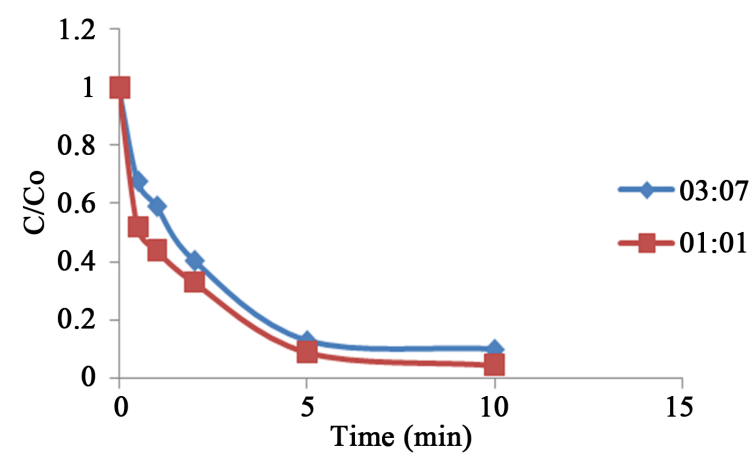

Figure 2. Effect of volume of internal aqueous phase on extraction of copper.

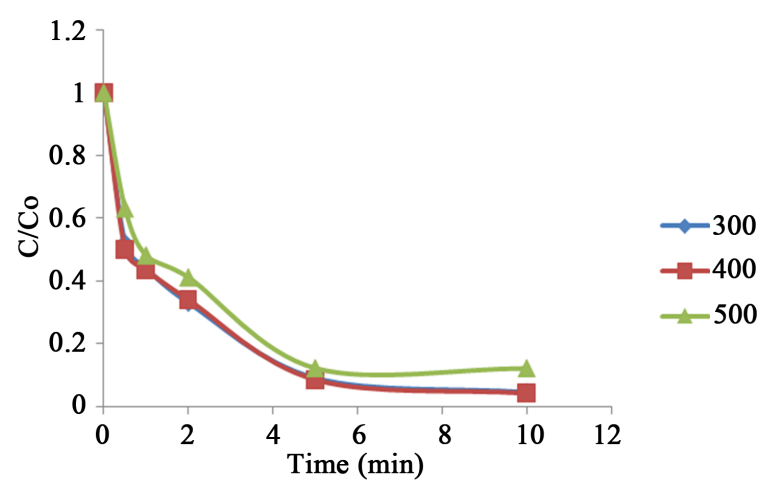

Figure 3. Effect of stirring speed on extraction of copper.

\subsection{Effect of Surfactant Concentration on Extraction of Copper}

The surfactant is probably the most important component in the emulsion liquid membrane technique, since a very stable primary emulsion, where no break-up of the internal droplets occurs is a prior condition for the application of the emulsion liquid membrane [4]. Surfactant concentration has influence on stability of emulsion and the extraction performance of copper. The surfactant used is Span 80 which is a commercial non-ionic surfactant with better stability than other surfactants and minimum resistance to mass transfer. It has other benefits as very low solubility in aqueous phase and can be easily demulsified.

Extraction experiments were conducted with varying concentrations of Span 80. As shown in Figure 4, with the surfactant concentration as $2 \%(\mathrm{v} / \mathrm{v})$ of Span 80 , the extraction\% was higher than with $3 \%$. Increased surfactant concentration leads to diffusional resistance as viscosity increases with surfactant concentration and decreases the diffusion coefficient and mass transfer coefficient. This decreases the transport of metal ions through the membrane phase. Also with increased surfactant ratio the subsequent demulsification and product recovery steps are difficult. Yan and Pal (2001) [5] have observed that swelling ratio generally increases with the increase in the concentration of surfactant present in the membrane (oil) phase. But at the same time with increased surfactant concentration, the breakage rate reduces giving a stable emulsion. Increase in surfactant concentration increases viscosity of membrane and improves emulsion stability [14].

With low surfactant concentration, membranes are prone to breakage thereby affecting the stability. But at low surfactant concentration due to low viscosity, the diffusion coefficient and the mass transfer coefficient will increase which will increase the extraction performance theoretically. Hence optimization has to be done for the breakage rate and mass transfer rate by taking the optimum amount of surfactant.

\subsection{Effect of Treat Ratio on Extraction of Copper}

Treat ratio is the ratio of volume of aqueous feed to that of emulsion. Change in treat ratio is brought by changing volume of feed and maintaining constant volume of emulsion phase. Treat ratio is kept in the range of 5:1 to 8:1. It is seen from Figure 5 that with increase in treat ratio, the percent extraction of copper decreases. At lower 


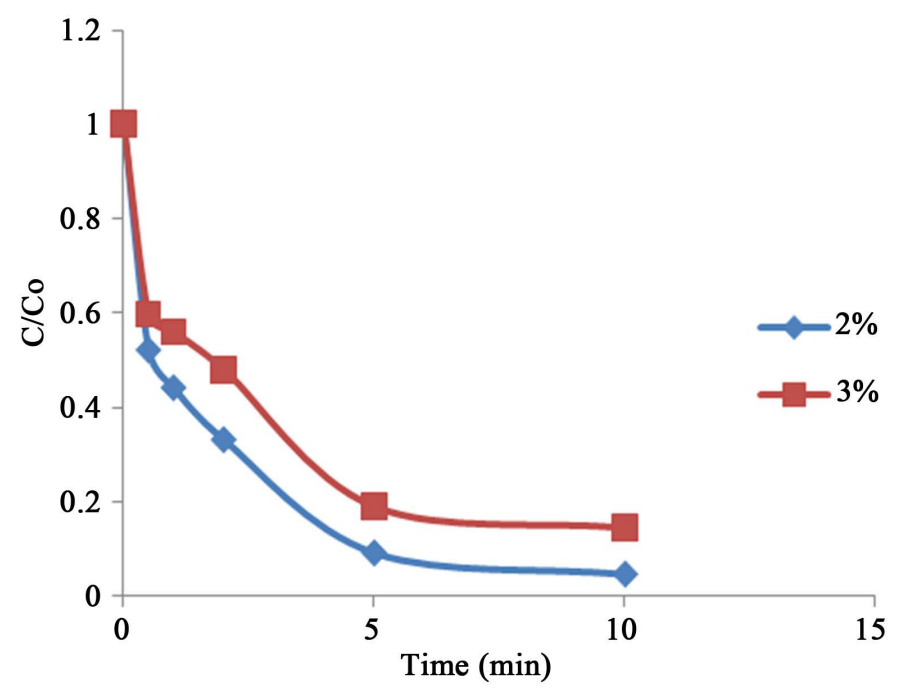

Figure 4. Effect of surfactant concentration on extraction of copper.

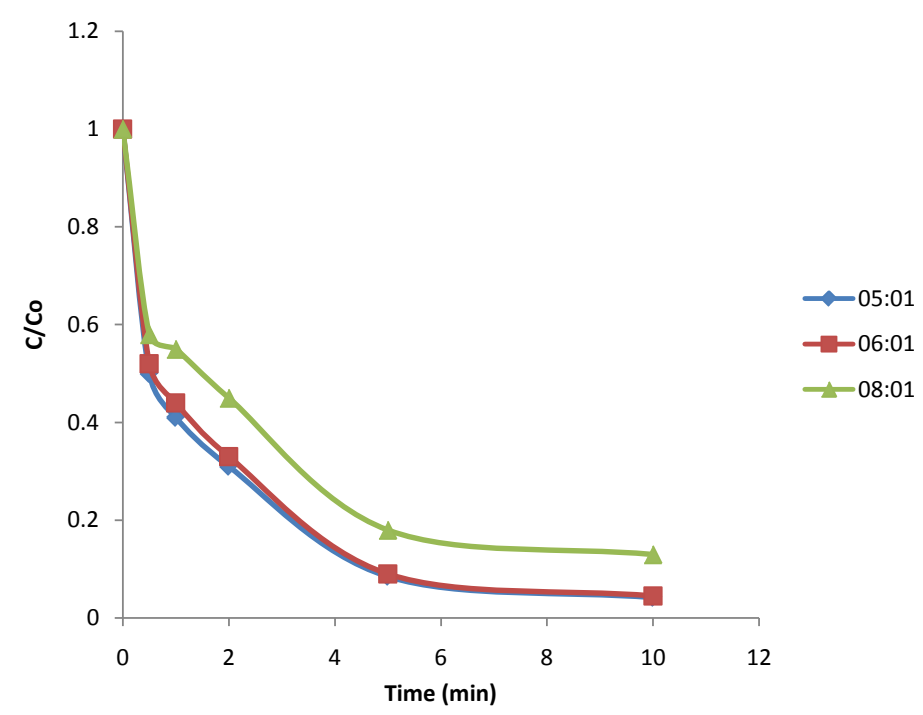

Figure 5. Effect of treat ratio on copper extraction.

treat ratio, the volume of emulsion as a whole is more with large surface area for more mass transfer. As a result higher degree of extraction is possible. With a higher treat ratio less emulsion is used for extraction which is desired from the point view of maximum enrichment of metal with respect to the feed phase. But the droplets do not contain enough internal reagents to react with the solute transported through the liquid membrane. As a result the extracted metal ions accumulate in the membrane phase and carrier does not diffuse back to react with the metal in the external aqueous phase.

Longer paths for diffusion of solute transport, hydrodynamic effect and stability of emulsion globules have a significant effect on extraction efficiency. Treat ratio of 5:1 was found to be a good value for high enrichment of copper in the strip phase.

\section{Conclusion}

The removal of copper from dilute solution by LEMs was studied using LIX 664N as the extractant, Span 80 as the surfactant and sulphuric acid as the stripping agent. It was found that maximum copper extraction was possible with $3 \% \mathrm{v} / \mathrm{v}$ surfactant, at $300 \mathrm{rpm}$ extraction speed with extraction time of 10 minutes with $\mathrm{pH}$ of feed as 2 and treat ratio of 6:1. The enrichment was 11.6 times. Thus LEMs can prove to be very useful for copper recov- 
ery from waste water where the metal concentrations are very low and can help in enrichment of very dilute streams.

\section{Acknowledgements}

We would like to thank the management of Mukesh Patel School of Technology Management \& Engineering (NMIMS) Mumbai for supporting the work. We thankfully acknowledge Mohini Organics for supplying Span 80 as a free sample and Mr. Manish Kapadia (BS International, Mumbai) for supplying LIX 664N.

\section{References}

[1] Frankenfeld, J.W., Cahn, R.P. and Li, N.N. (1981) Extraction of Copper by Liquid Membranes. Separation Science and Technology, 16, 385-402. http://dx.doi.org/10.1080/01496398108068528

[2] Hayworth, H.C., Ho, W.S., Burns Jr., W.A. and Li, N.N. (1983) Extraction of Uranium from Wet Process Phosphoric Acid by Liquid Membranes. Separation Science and Technology, 18, 493-521. http://dx.doi.org/10.1080/01496398308060291

[3] Draxler, J. and Marr, R. (1986) Emulsion Liquid Mebranes Part I: Phenomenon and Industrial Application. Chemical Engineering and Processing, 20, 319-329. http://dx.doi.org/10.1016/0255-2701(86)80010-1

[4] Draxler, J., Furst, W. and Marr, R. (1988) Separation of Metal Species by Emulsion Liquid Membranes. Journal of Membrane Science, 38, 281-293. http://dx.doi.org/10.1016/S0376-7388(00)82425-4

[5] Yan, J. and Pal, R. (2001) Osmotic Swelling Behaviour of Globules of W/O/W Emulsion Liquid Membranes Membranes. Journal of Membrane Science, 190, 79-91. http://dx.doi.org/10.1016/S0376-7388(01)00424-0

[6] Kulkarni, P.S., Tiwari, K.K. and Mahajani, V.V. (1999) Studies in Extraction of Nickel by Liquid Emulsion Membrane Process. Indian Journal of Chemical Technology, 6, 329-335.

[7] Kankekar, P.S., Wagh, S.S. and Mahajani, V.V. (2010) Process Intensification in Extraction by Liquid Emulsion Membrane (LEM) Process: A Case Study; Enrichment of Ruthenium from Lean Aqueous Solution. Chemical Engineering and Processing, 49, 441-448. http://dx.doi.org/10.1016/j.cep.2010.02.005

[8] Kankekar, P.S., Wagh, S.S. and Mahajani, V.V. (2010) Process Intensificatin via Liquid Emulsion Membrane Technique in Extraction and Enrichment of Rhodium (III) from Chloride Media. Separation Science and Technology, 45, 562-571. http://dx.doi.org/10.1080/01496390903526410

[9] Sengupta, B., Sengupta, R. and Subrahmanyam, N. (2006) Copper Extraction into Emulsion Liquid Membranes Using LIX 984N-C. Hydrometallurgy, 81, 67-73. http://dx.doi.org/10.1016/j.hydromet.2005.10.002

[10] Mohamed, Y.T. and Ibrahim, A.H. (2012) Extraction of Copper from Waste Solution Using Liquid Emulsion Membrane. Journal of Environmental Protection, 3, 129-134. http://dx.doi.org/10.4236/jep.2012.31016

[11] Sengupta, B., Sengupta, R. and Subrahmanyam, N. (2006) Process Intensification of Copper Extraction Using Emulsion Liquid Membranes: Experimental Search for Optimal Conditions. Hydrometallurgy, 84, 43-53. http://dx.doi.org/10.1016/j.hydromet.2006.04.002

[12] Kumar, R., Shah, D.J. and Tiwari, K.K. (2013) Separation of Copper and Nickel by Solvent Extraction Using LIX 664N. Journal of Environmental Protection, 4, 315-318. http://dx.doi.org/10.4236/jep.2013.44037

[13] Kulkarni, P.S., Tiwari, K.K. and Mahajani, V.V. (2000) Membrane Stability and Enrichment of Nickel in Liquid Emulsion Membrane Process. Journal of Chemical Technology \& Biotechnology, 75, 553-560. http://dx.doi.org/10.1002/1097-4660(200007)75:7<553::AID-JCTB252>3.0.CO;2-I

[14] Reis, M.T.A. and Carvalho, J.M.R. (1993) Recovery of Zinc from Industrial Effluent by Emulsion Liquid Membranes. Journal of Membrane Science, 84, 201-211. http://dx.doi.org/10.1016/0376-7388(93)80016-Q 
Scientific Research Publishing (SCIRP) is one of the largest Open Access journal publishers. It is currently publishing more than 200 open access, online, peer-reviewed journals covering a wide range of academic disciplines. SCIRP serves the worldwide academic communities and contributes to the progress and application of science with its publication.

Other selected journals from SCIRP are listed as below. Submit your manuscript to us via either submit@scirp.org or Online Submission Portal.
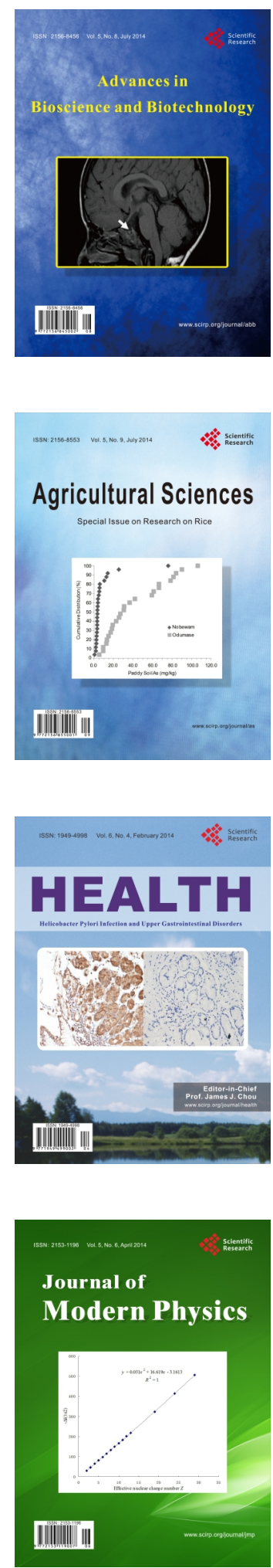
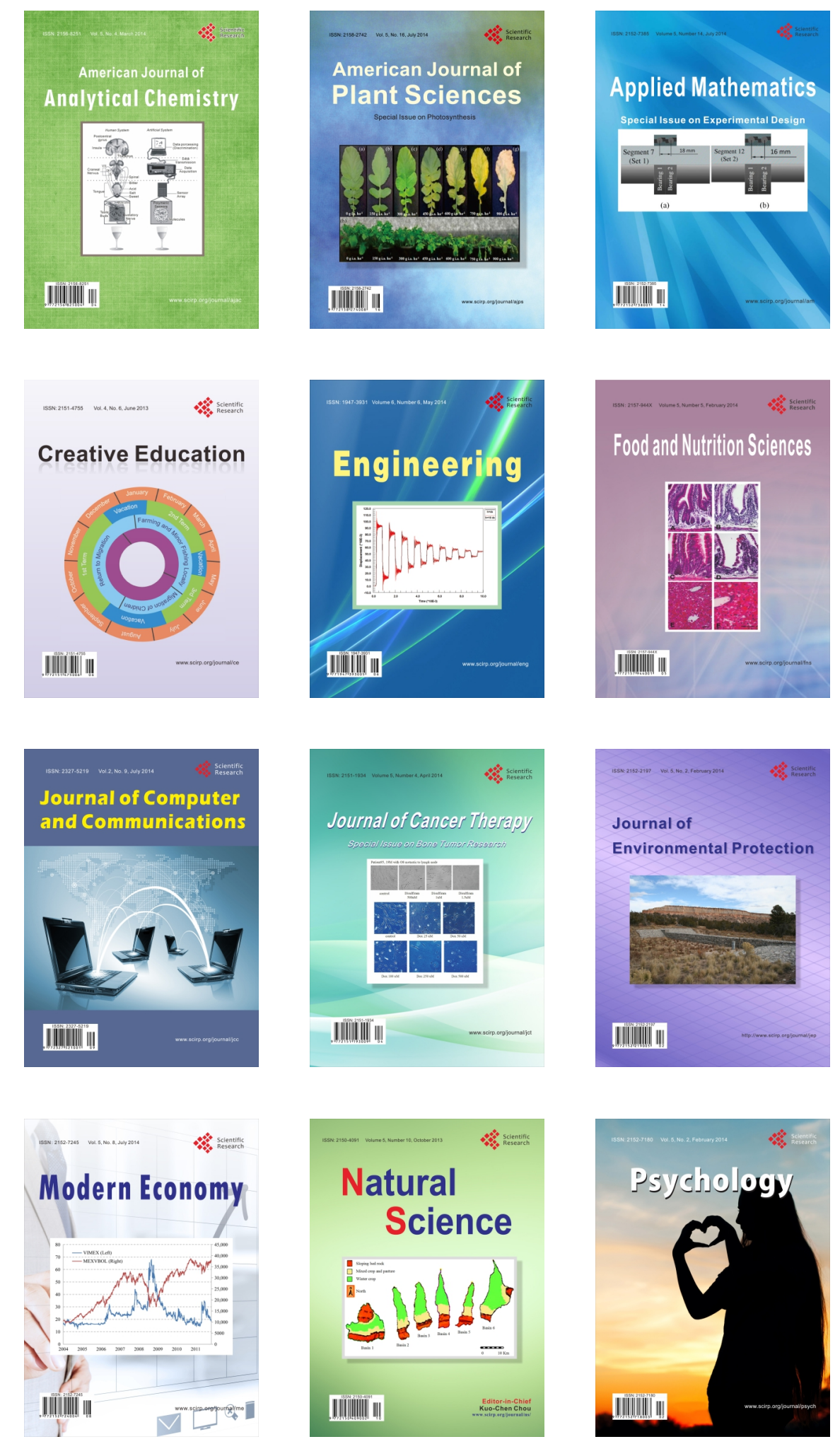